\title{
Cutoff rigidity and particle trajectories online calculator
}

\author{
Semen M. Belov, Egor Zobnin, Victor G. Yanke \\ Correspondence \\ Pushkov Institute of Terrestrial Magnetism, Ionosphere and Radio Wave Propagation (IZMIRAN), Moscow, Russia, \\ sfrovis@gmail.com, egorizzee@gmail.com,yanke@izmiran.ru
}

\section{OPEN ACCESS}

This work is published under the Creative Commons Attribution 4.0 International licence (CC BY 4.0) Please note that individual, approPlease note that individual, appropriately marked parts of the work may be excluded from the licence mentioned or may be subje other copyright conditions.
If such thirdparty material is no If such thirdparty material is not
under the Creative Commons license any copying, editing or public reproduction is only permitted with the prior consent of the respective copyright owner or on the basis of relevant legal authorization regulations.

\section{Keywords}

magnetospheric effect; cutoff rigidity; particle trajectories; cosmic rays

\begin{abstract}
Over the years, many authors have developed unique software packages for calculating the geomagnetic cutoff rigidities and the asymptotic directions of particle arrival. Such programs are used for mass calculations and require some skill. However, it is often necessary to carry out single calculations with the same accuracy. For this purpose, calculator programs have been created on the basis of already developed software packages. One of such programs, a calculator, is described in this work.
\end{abstract}

\section{Introduction}

The magnetospheric effect of cosmic rays, i.e. the change in particle flux in response to a change in the state of the magnetosphere or position in the magnetosphere was discovered by Clay in 1927, who revealed the latitudinal effect on the Amsterdam-Sydney route and used the Earth's magnetic field as a charged particle spectrometer for the first time. The correct explanation of the Clay effect was given in (Bothe \& Kolhorster 1929) in assuming that some of the cosmic rays must be charged particles.

Bruno Rossi (1930) predicted the difference between the intensities of cosmic rays coming from the east and west. It was found that the intensity is higher from the western directions, which indicates that most of the primary particles are positive.

The first who appreciated the extreme importance for space physics of the problem of the motion of charged particles in the Earth's magnetic field was Størmer (1930). In the dipole approximation, Størmer obtained an analytical expression for the trajectories of cosmic rays (albeit only in the equatorial plane) and introduced the concept of forbidden trajectories.

Lemaitre and Vallarta (1936a, 1936b), developing Størmer's theory and the concept of a forbidden cone, introduced the concept of a permitted cone when all trajectories are permitted, and the concept of a basic cone, which includes a permitted cone and a penumbra. At the equator, the permitted and main cones coincide, but at mid-latitudes, there are sufficient differences: the region of rigidity between the cones forms penumbra, i.e. many allowed and forbidden trajectories. The relative role of the penumbra increases with increasing geomagnetic latitude and completely disappears at high latitudes. The area of the penumbra was directly discovered by them when calculating the trajectories of particles in a dipole field on analog computers.

Jory (1956) was the first to carry out numerical trajectory calculations of cosmic rays in a dipole magnetic field. McCracken (McCracken et al. 1962) carried out numerical trajectory calculations of cosmic rays already in a real geomagnetic field, represented by six spherical harmonics. 
Two key concepts, such as the geomagnetic cutoff rigidity (for describing isotropic variations) and the asymptotic cone of particle acceptance (for describing anisotropic variations), allow us to describe all magnetospheric effects of cosmic rays. When describing long-term variations, it is sufficient to restrict ourselves to the isotropic approximation. At present, the method of numerical integration of the equations of motion of charged particles is generally accepted for determining the cutoff rigidities. With this method, the cutoff rigidity can be calculated with any precision. Moreover, when approximating the geomagnetic field, both internal and external sources of the magnetic field can be taken into account.

The most complete and systematic studies of the magnetospheric effects of cosmic rays, including their long-term changes, were carried out by M. Shea and D. Smart based on the software package (Cutoff Rigidity Program 2020). The global distributions of the vertical rigidity of the geomagnetic cutoff were calculated with a step of $5^{\circ} \times 15^{\circ}$ in latitude and longitude for the epochs from 1955 to 2000 (Smart \& Shea, 2007a, 2007b). Vertical geomagnetic cutoff rigidities were obtained for all stations of the global network for ten five-year epochs 1955-2000 (Shea \& Smart 1965, 1966, 1967; Smart \& Shea 1994, 2001, 2003). In Shea \& Smart 1975 and Storini et al. 1999, the unevenness of changes in the planetary distribution of geomagnetic cutoff rigidities over 20 years from 1955 to 1975, especially in the southern Atlantic Ocean, is shown. The South Atlantic saw a decrease in rigidity, while the North showed a comparable increase in vertical cutoff rigidity.

In Gvozdevsky et al. 2016, for the vertical directions of particle arrival by the method of trajectory calculations, a planetary distribution of the geomagnetic cutoff rigidities with a step of $5^{\circ} \times 15^{\circ}$ in latitude and longitude was obtained for the period 1950-2020 with a forecast up to 2050 . For the period 1950-2050, temporal variations of the geomagnetic cutoff rigidities were also obtained for the worldwide network of neutron monitors. From the data of muon telescopes, the geomagnetic cutoff rigidity of inclined directions of particle arrival was also obtained.

The purpose of this work is to create a calculator for obtaining the geomagnetic cutoff rigidity with the most complete graphical presentation of the results. To carry out trajectory calculations, use the developed and debugged software packages.

\section{Method of trajectory calculations}

The main method for studying the trajectories of charged particles in the geomagnetic field is the numerical integration of the equation of motion. At present, the most widespread use of various modifications of the 4th order Runge-Kutta method, with the use of standard methods of accuracy control, including checking the conservation of the integral of motion - energy (or modulus of velocity). In addition, the reverse integration method is used to check the correctness of the numerical solution. Its essence is that due to the structure of the Lorentz equation, which describes the motion of a charged particle in a stationary magnetic field, with the simultaneous replacement of the sign of the particle charge and its velocity vector with opposite values, the trajectory of motion is completely preserved, but passes in the opposite direction. Having stopped at some point the numerical integration, one can try to return to the starting point (or in its vicinity) by backward integration, thereby estimating the error of the numerical solution. The same method of backward integration is used to determine the initial point at which a cosmic ray particle came from interplanetary space to the boundary of the Earth's magnetosphere. Since the main flux of cosmic rays is made up of protons, the calculations use a proton with the opposite sign.

Integration ends in three cases:

- either after a specified time has elapsed (the particle is considered captured),

- either the particle crossed the magnetopause surface (went beyond the magnetosphere),

- or its radius vector turned out to be less than $\left(\mathrm{R}_{\mathrm{E}}+20\right) \mathrm{km}$ (the particle returned to the atmosphere). 
In the case of the second result, this trajectory is marked as allowed, otherwise, it is prohibited. The described method of trajectory calculations was first formulated and applied in McCracken et al. (1962) and is also described in Smart \& Shea (2001). This technique is implemented in works (Gvozdevsky et al. 2015, 2016, 2017, 2018, 2019). Particular attention was paid to the issue of accounting for the penumbra. The results are archived on the server (Mag_Effect 2018).

\section{Involved models of the magnetosphere}

For the trajectory calculations, the model of the International Geomagnetic Reference Field IGRF-12 (Thebault et al. 2015) was used. This is a model of the main geomagnetic field for the 2015.0 epoch and a linear annual predictive model of the secular variation for 2015.0-2020.0. We have continued this linear approximation of the coefficients of the Gaussian expansion until 2050. The geomagnetic field model is represented by the 13th spherical harmonics, but the predictive model is limited to the 8th harmonics. For trial calculations of the characteristics of the magnetic field at different points of the magnetosphere, one can use a calculator (Magnetic calculator 2019).

The contribution of external magnetic fields was taken into account based on the empirical models of Tsyganenko (Magnetospheric modeling 2020). To describe the contribution of external magnetic fields to quiet or weakly disturbed periods (from $\mathrm{Kp}=0$ to $\mathrm{Kp}=5$ ), the widely used Tsyganenko T89 model was used (Tsyganenko 1989). At higher disturbances, it is necessary to use the T96 model (Tsyganenko 1996), which describes the position of the magnetopause depending on the state of the solar wind. The input parameters in the T96 model are the density and speed of the solar wind, which determine its pressure on the magnetosphere, the By and Bz components of the IMF, and the Dst index of geomagnetic activity. If periods of a very strongly disturbed magnetosphere (Dst $\leq-65 \mathrm{nT}$ ) are considered, then the Ts02 (Tsyganenko 2003) or Ts04 (Tsyganenko 2005, 2013) models should be involved, which differ in different approximations of the same experimental data.

\section{Description of the program calculator Cutoff-2050}

The Cutoff-2050 Calculator is available at https://tools.izmiran.ru/ (Calculator Cutoff-2050, 2020). The calculator for calculating geomagnetic cutoff rigidities is based on the Cutoff-2050 program, which was used in (Gvozdevsky et al. 2019 and references). Cutoff-2050 program is located at the same address.

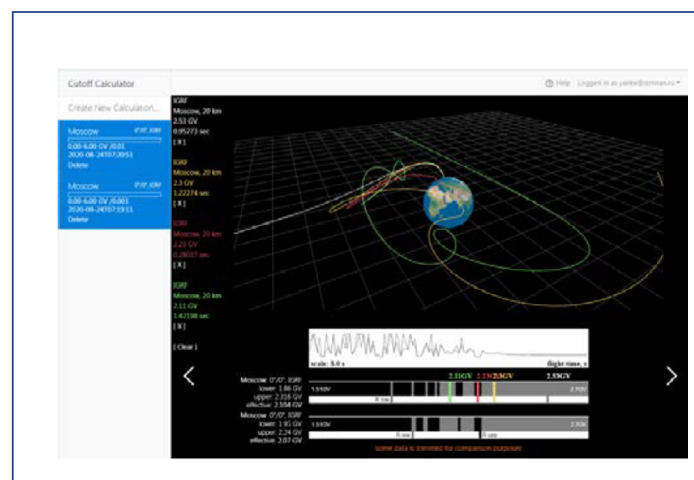

Figure 1: Comparison of the vertical geomagnetic cutoff rigidity at Moscow station obtained with different steps of integration: 0.01 and $0.001 \mathrm{GV}$.

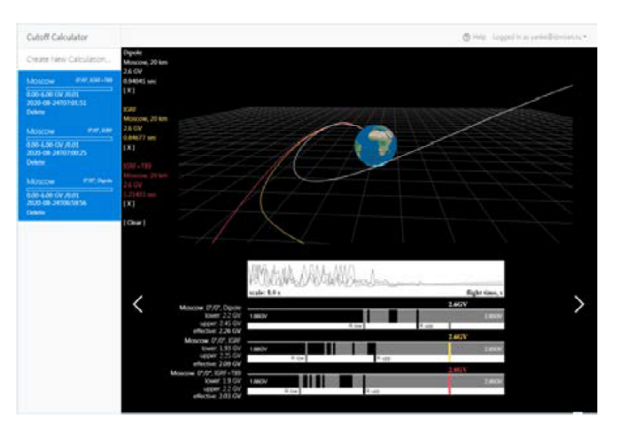

Figure 2: Comparison of the vertical geomagnetic cutoff rigidity at Moscow station obtained with for the models: Dipole, IGRF, IGRF+T89 and integrating step $0.01 \mathrm{GV}$. 
At the first login, any user creates a personal account, in which he can accumulate and compare the results of calculations. When you select Create New Calculation, a dialog box of parameters appears, the values of which are obvious.

So, for example, figure 1 shows the calculations and comparison of the vertical rigidity of the geomagnetic cutoff for the Moscow station for two values of the integration steps. If you select the hardness of a particle on the penumbra scale with the mouse, then the trajectory of this particle in this magnetic field is displayed. Above the penumbra field, there is a graph showing the time of particle motion until it leaves the magnetosphere for each rigidity in the considered range.

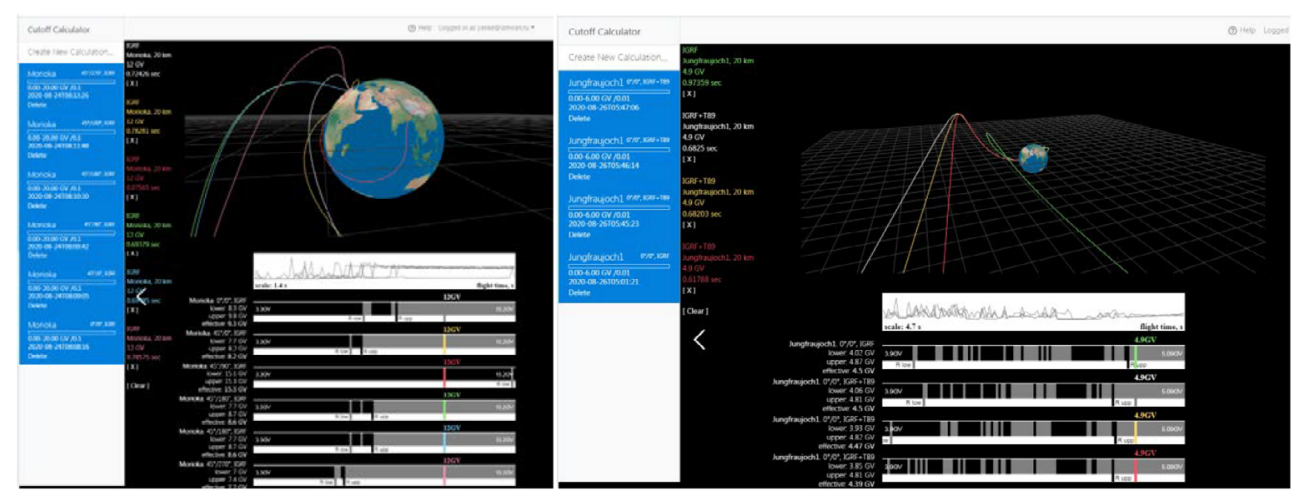

Figure 3: Geomagnetic cutoff rigidity for Morioka under

Figure 4: Geomagnetic cutoff rigidity for Jungfraujoch model IGRF+T89 and integrating step $0.1 \mathrm{GV}$ for vertical, zenith angle $45^{\circ}$ and azimuth angles $0^{\circ}, 90^{\circ}, 180^{\circ}, 270^{\circ}$. station at the models IGRF and IGRF+T89 $(K p=0,2,4)$.

Figure 2 shows another example, which compares the results obtained using three magnetic field models: dipole, IGRF, IGRF + T89. Particle trajectories are also displayed after selecting the appropriate stiffness on the penumbra scale. Three trajectories for $2.6 \mathrm{GV}$ particles are compared.

Figure 3 shows an example of vertical and inclined trajectories of particle motion using the Morioka telescope for the IGRF model of the magnetic field. Particle trajectories are also displayed after selecting the appropriate rigidity on the penumbra scale. The trajectories are compared for particles with a rigidity of $12.0 \mathrm{GV}$.

Figure 4 compares the geomagnetic cutoff rigidities for Jungfraujoch for the IGRF and IGRF + T89 models $(\mathrm{Kp}=0,2,4)$.

Table 1 shows the parameters of the test calculations of the calculator for various models. Calculating the geomagnetic cutoff rigidities for the IGRF + T96 model and especially for the IGRF + T02 model requires a lot of computational time and this must be taken into account when planning the calculations.

\begin{tabular}{|c|c|c|c|c|c|c|c|c|c|}
\hline \multirow[t]{3}{*}{ Model } & \multirow{2}{*}{\multicolumn{5}{|c|}{ Parametrs }} & \multicolumn{4}{|l|}{ Result } \\
\hline & & & & & & \multicolumn{3}{|c|}{ Cutoff rigidities, GV } & \multirow{2}{*}{$\begin{array}{l}\text { Calculation } \\
\text { time, sec }\end{array}$} \\
\hline & Kp & $\begin{array}{l}\mathrm{P}_{\text {sw }}(\mathrm{nPa}) \\
\text { Solar wind } \\
\text { dynamic } \\
\text { pressure }\end{array}$ & $\begin{array}{l}D_{\text {st-index }} \\
(n T)\end{array}$ & $\begin{array}{l}\text { IMF } \\
\mathrm{B}_{\mathrm{y}} \& \mathrm{~B}_{\mathrm{z}} \\
(\mathrm{nT})\end{array}$ & $\mathrm{G}_{1} \& \mathrm{G}_{2}$ & lower & upper & effective & \\
\hline Dipole & & & & & & 2.337 & 2.737 & 2.498 & 6 \\
\hline IGRF & & & & & & 1.765 & 2.416 & 2.185 & 37 \\
\hline IGRF+T89 & 4 & & & & & 1.582 & 2.190 & 1.922 & 28 \\
\hline IGRF+T96 & & 2 & -50.0 & 0 and 5 & & 1.518 & 2.156 & 1.906 & 1188 \\
\hline IGRF+TsO2 & & 2 & -50.0 & 0 and 5 & 6 and 10 & 1.649 & 2.234 & 1.947 & 3964 \\
\hline
\end{tabular}

rigidity lower and upper limit (GV): $0.0-3.0$

step $0.001 \mathrm{GV}$, Maximum time of flight - $180.00 \mathrm{~s}$.

Table 1: For 01.07.2017 00:00:00 and Geographic coordinates (latitude, longitude) $(55.00,40.00)$, Vertical angle 0.00 and Azimuthal angle 0.00 . 


\section{Available calculators for calculating geomagnetic cutoff rigidities}

Several calculators have been developed for calculating geomagnetic cutoff rigidities. A simple online internet project (Cutoff rigidity Calculator 2010) is based on the IGRF model and considers only vertically falling particles. The algorithm described in (Smart \& Shea 2001) is applied. Disadvantages: the contribution of the outer magnetosphere is not taken into account, only vertical trajectories are considered, there is no graphical presentation of the results of calculating the penumbra, trajectories.

Another online internet project (Web Calculators 2019) is based on more complex magnetosphere models (Tsyganenko 1996; Tsyganenko et al. 2005) with automatic involving of the input parameters of the interplanetary medium necessary for the magnetosphere model, which is a very convenient and useful option. The program also reconstructs the trajectories of particles inside the magnetosphere. The calculator has a number of other useful features like calculating the Larmor radius inside, R-E converter. Disadvantages: only vertical trajectories are considered, there is no graphical presentation of the results of trajectory calculations - penumbra.

The online calculator (Calculator cutoff 2018) is based on the methodology described in (Gvozdevsky et al. 2019 and references). The result of the calculator's work is the lower, upper and effective values of the geomagnetic cutoff and penumbra stiffness for one of the specified magnetosphere models: dipole, IGRF, Tsyganenko IGRF + T89, IGRF + T96, IGRF + T02 models. Disadvantages: no graphical representation of trajectories.

\section{Conclusions}

1). The Cutoff-2050 calculator calculates the rigidity of the geomagnetic cutoff and particle trajectory for a given date (1900-2050) and at a given geographic point for magnetosphere models: dipole, IGRF, Tsyganenko model: IGRF + T89 and higher order IGRF + T96 and IGRF + models T02, but the latter require a long calculation time.

2). For regular users, it is possible to organize on the server personal accounts of users to accumulate and store the results obtained.

3). Visualization of all the results obtained: penumbra, time of movement and trajectories of particles.

4). The calculator interface allows you to visually carry out a comparative analysis of trajectories for various models and parameters.

5). The Cutoff-2050 calculator is an effective tool for urgent single calculations.

6). The task has significant educational potential since the capabilities of the online calculator can be used to teach students of the relevant specialties.

\section{Acknowledgments}

This work was partially supported by the grant RFBR No. 18-02-00451. Experimentally and methodologically support the project USU „Russian national network of ground stations of cosmic rays“. We are grateful to all the staff of the World Network of cosmic ray stations http://cr0.izmiran.ru/ ThankYou.

\section{References}

Bothe W., Kolhorster W., 1929, Das Wesen der Höhenstrahlung, Ztschr. Phys., Bd. 56, S. 751-777

Cooke, D. J., Humble J. E., Shea M. A., Smart D. F., et al. 1991, "On Cosmic-Ray Cut-Off Terminology" Il Nuovo Cimento C14, 213-234, D0I: https://doi.org/10.1007/BF02509357

Gvozdevsky, B., Dorman L., Abunin, A., Preobrazhensky, M., Gushchina, R., Belov, A., Eroshenko, E.,Yanke, V., 2015, "Variations of the vertical 
cut off rigidities for the world wide neutron monitor network over the period of continues monitoring of cosmic rays", Proc. 34th ICRC, Hague, https://pos.sissa.it/236/203/pdf (last accessed April 9, 2021)

Gvozdevsky, B. B., Abunin, A., Kobelev, P. G., Gushchina, R. T., Belov, A. V., Eroshenko. E. A., Yanke, V. G., 2016, Magnetospheric effects of cosmic rays. I. Long-term changes in the geomagnetic cutoff rigidities for the stations of the global network of neutron monitors, Geomagnetism and aeronomy: V. 56, No 4, 381-392, DOI: https://doi.org/10.1134/S0016793216040046

Gvozdevsky, B., Belov, A., Gushchina, R., Eroshenko, E., Preobrazhensky, M., Yanke, V., 2017, "The secular variations of cosmic ray cutof rigidities, caused by century variations in geomagnetic field, and cosmic ray variations", 35th International Cosmic Ray Conference ICRC 2017, 10-20 July, 2017, Bexco, Busan, Korea, https://pos.sissa.it, PoS(ICRC2017)067, 12-20, https://pos.sissa.it/301/067/pdf (last accessed April 9, 2021)

Gvozdevsky, B. B., Belov, A. V., Gushchina, R. T., Eroshenko, E. A., Kobelev, P. G., Yanke, V. G., 2017, Long-term changes in the vertical rigidity of the geomagnetic cutoff over the entire period of monitoring of cosmic rays, „Physics of Auroral Phenomena" Proc. XL Annual Seminar, Apatity, 89-93, http://pgia.ru:81/seminar/archive (last accessed April 9, 2021)

Gvozdevsky, B. B., Belov, A. V., Gushchina, R. T., Eroshenko, E. A., Danilova, O. A., Yanke, V. G., 2018a, Peculiarities of long-term changes in the rigidity of geomagnetic cutoff of cosmic rays of inclined directions, "Physics of Auroral Phenomena" Proc. 41st Annual Seminar, Apatity, 80-83, DOI: https://doi.org/10.25702/KSC.2588-0039.2018.41.80-83

Gvozdevsky, B. B., Belov, A.V., Gushchina, R.T., Kobelev, P.G., Eroshenko, E.A., Yanke, V.G., 2018b, "Long-Term Changes in Vertical Geomagnetic Cutoff Rigidities of Cosmic Rays", Physics of Atomic Nuclei, Vol. 81, No. 9, 1382-1389, D0I: https://doi.org/10.1134/ S1063778818090132

Gvozdevsky, B. B., Belov, A. V., Gushchina, R.T., Eroshenko, E.A., Yanke, V.G., 2019, "Planetary long term changes of the cosmic ray geomagnetic cut off rigidities" (26th Extended ECRS + 35th RCRC, Barnaul, Russia, 6-10 July 2018. SH34). Journal of Physics: Conference Series, 012008, DOI: https://doi.org/10.1088/1742-6596/1181/1/012008

Jory, F.S., 1956, Selected cosmic-ray orbits in the Earth's magnetic field, Phys. Rev. V.103. № 4., 1068-1075

Lemaitre, G.E., Vallarta, M.S., 1936, On the Geomagnetic Analysis of Cosmic Radiation, Phys. Rev. V. 49, 719-726

Lemaitre, G. E., Vallarta, M.S., 1936a, On the Allowed Cone of Cosmic Radiation, Phys. Rev. V.50, 493-504

McCracken, K.G., Rao, U.R., Shea, M.A., 1962, The Trajectories of Cosmic Rays in a High Degree Simulation of the Geomagnetic Field, M.I.T. Techn. Rep. 77, Lab. for Nucl. Sci. and Eng., Mass. Inst. of Technol., Cambridge

Rossi, B., 1930, "Method of Registrating Multiple Simultaneous Impuls of Several Geigers's Counters", Nature, 125, No. 3156, 636-636, D0I: https://doi.org/10.1038/125636a0

Shea, M.A., Smart, D.F., McCracken K.G., 1965, A study of vertical cutoff rigidities using sixth degree simulations of the geomagnetic field, J.Geophys.Res., V. 70. - N 17. - P.4117-4130

Shea, M.A., Smart, D.F., 1966, "Vertical cutoff rigidities in the South Atlantic", Space Res., 6, 177-187

Shea, M.A., Smart, D.F., 1967, "Worldwide trajectory-derived vertical cutoff rigidities and their application to experimental measurements for 1955", JGR, V72, No7, 2021-2028

Shea, M.A., Smart, D.F., 1975, "A five by fifteen degree world grid of calculated cosmic ray vertical cutoff rigidities for 1965 and 1975 ", Proc. 14th Int. Cosmic Ray Conf., Munchen, 4, 1298-1303

Smart, D.F., Shea, M.A., 1994, "Geomagnetic cutoffs: A review for space dosimetry applications", Adv. Space Res., 14, 10, 787-796

Smart, D. F., Shea, M. A., 2001, Geomagnetic Cutoff Rigidity Computer Program: Theory, Software Description and Example, NASA Technical Reports Serve, Final Report, 199 pp., 18 January 2001, ID: 20010071975 (last accessed April 9, 2021)

Smart, D.F., Shea, M.A., 2003, "The space developed dynamic vertical cutoff and its applicability to aircraft radiation dose", Adv. Space Res., $32,1,103-108$

Smart, D.F., Shea, M.A., 2007a, "World Grid of Calculated Cosmic Ray Vertical Cutoff Rigidities for Epoch 1995.0", Proc. 30th ICRC, Mexico, V.1 (SH), 733-736

Smart, D.F., Shea, M.A., 2007b, "World Grid of Calculated Cosmic Ray Vertical Cutoff Rigidities for Epoch 2000.0", Proc. 30th ICRC, Mexico, V.1 (SH), 737-740

Storini, M., Shea, M.A., Smart, D.F., Cordaro, E.G., 1999, "Cuttof variability for Antarctic Laboratory for Cosmic Rays (LARC:1955-1995)", 26th ICR, Salk Lake City, SH3.6.30 (last accessed April 9, 2021), Vol. 7, 402-405,.

Størmer, C., 1930, On the trajectories of electric particles in the field of magnetic dipole with applications to the theory of cosmic radiation Astrophysics. 1:237

Thebault, E., Finlay, C. C., Zvereva, T,et al 2015, "International Geomagnetic Reference Field: the 12th generation", Earth, Planets and Space, V 67, No 79, DOI: https://doi.org/10.1186/s40623-015-0228-9

Tsyganenko, N. A., 1989, A magnetospheric magnetic field model with a warped tail current sheet, Planet. Space Sci., 37, No. 1, 5-20, D0I: https://doi.org/10.1016/0032-0633(89)90066-4

Tsyganenko N. A., Stern, D. P., 1996, Modeling the global magnetic field of the large-scale Birkeland current systems, J. Geophys. Res. 101, 27187-27198, DOI: https://doi.org/10.1029/98JA02292

Tsyganenko, N. A., Singer, H. J., Kasper, J. C., 2003, Storm-time distortion of the inner magnetosphere: How severe can it get?, J. Geophys. Res., 108, No. A5, 1209, DOI: https://doi.org/10.1029/2002JA009808

Tsyganenko, N. A., Sitnov, M. I., 2005, Modeling the dynamics of the inner magnetosphere during strong geomagnetic storms, J. Geophys. Res. 110, A03208, DOI: https://doi.org/10.1029/2004JA010798

Tsyganenko, N.A., 2013, Data-based modelling of the Earth's dynamic magnetosphere: a review, Ann. Geophys., 31, 1745-1772, D0I: https://doi.org/10.5194/angeo-31-1745-2013

\section{Online data products}

Calculator CutOff-2050, 2020: https://tools.izmiran.ru (last accessed April 9, 2021)

Cutoff rigidity Calculator, 2010, Zreda Marek, COSMOS project University of Arizona, http://cosmos.hwr.arizona.edu/Util/rigidity.php (last accessed April 9, 2021)

Cutoff Rigidity Program, 2020 https://ccmc.gsfc.nasa.gov/modelweb/sun/cutoff.html (last accessed April 9, 2021)

Magnetic calculator, 2019, https://geomag.nrcan.gc.ca/calc/calc-en.php (last accessed April 9, 2021) 
Magnetospheric modeling, 2020, http://geo.phys.spbu.ru/magmodel/empiric.html\#t13 (last accessed April 9, 2021)

Mag_Effect, 2018, http://cosrays.izmiran.ru/dbs/MagEffect (last accessed May 31, 2021)

Web Calculators, 2019, Boschini M. J., Della T. S., Gervasi M., Grandi D., Rancoita P. G. , Bobik P., Kudela K., http://www.geomagsphere.org (last accessed April 9, 2021)

\section{Questions and answers}

Monica Laurenza: Is it possible for the user to change all model parameters?

Answer: Yes, it is possible. But it should be remembered that the computation time for IGRF + T96 model, for example, and higher models can be quite long.

Alexander Mishev: What is the rigidity resolution for the computations $0.01,0.001 \mathrm{Gv}$ or less?

Answer: The integration step is especially important in the penumbra area. An integration step of 0.01 results in a rigidity error of $\sim 0.1 \mathrm{GV}$, an integration step of $\sim 0.001$ leads to a rigidity error of $\sim 0.05 \mathrm{GV}$.

Question: Is it possible to use the program on a PC?

Answer: Yes, it is possible.

Christian T. Steigies: IGRF has been updated recently (2019?), are you using the latest model?

Answer: No, this version was not involved. The IGRF-2015 model was used with a forecast up to IGRF-2020. But there are no problems with the inclusion of IGRF-2019.

James Ryan: Can this be adapted to low Earth orbiting spacecraft, like PAMELA?

Answer: Yes, the calculations are applicable across the entire magnetosphere.

Marc Duldig: Historically please remember the first paper to comprehensively discuss the CR penumbra: Cooke D. J., Humble J. E., Shea M. A., Smart D. F., et al. "On Cosmic-Ray Cut-Off Terminology" II Nuovo Cimento C14, 213-234 1991, DOI: https://doi.org/10.1007/BF02509357

Answer: Yes, thank you, the historical overview and helpful discussion of terminology should be preserved in our work.

Rolf Bütikofer: Do you plan to offer the determination of cutoff rigidities for a network with a selected mesh size in a single run?

Answer: No, we did not plan, but we shall plan, if necessary. The server http://tools.izmiran.ru contains tables $\left(5^{\circ} \times 15^{\circ} \mathrm{grid}\right)$ of planetary distributions of geomagnetic cutoff rigidities from 1900 to 2050 with a step of 5 years. But for a list of stations, such calculations can be organized.

Marc Duldig: Will you optimize using parallel processing with PC GPU?

Answer: No, until not. 